\title{
Ramucirumab for the treatment of patients with gastric or gastroesophageal junction cancer in community oncology practices
}

\author{
A. Scott Paulson ${ }^{1} \cdot$ Lisa M. Hess ${ }^{2} \cdot$ Astra M. Liepa $^{2} \cdot$ Zhanglin Lin Cui $^{2} \cdot$ Kathleen M. Aguilar $^{3} \cdot$ Jamyia Clark $^{3}$. \\ William Schelman ${ }^{2}$
}

Received: 3 October 2017 / Accepted: 14 January 2018 / Published online: 3 February 2018

(c) The International Gastric Cancer Association and The Japanese Gastric Cancer Association 2018

\begin{abstract}
Background Limited real-world research has investigated ramucirumab for the treatment of patients with gastric or gastroesophageal junction (GEJ) cancer. This study was designed to describe ramucirumab monotherapy or combination therapy use in a community oncology practice setting.

Methods This was a retrospective observational cohort study to describe the treatment of adult patients with gastric or GEJ cancer who initiated ramucirumab treatment between 4/21/14 and 6/30/16 within the US Oncology Network. Kaplan-Meier method and Cox proportional hazards regression analyses were used to assess clinical outcomes. Multivariable logistic regression models were used to assess patient-level predictors of ramucirumab monotherapy or combination therapy.

Results A total of 505 patients (mean age 64.4 years; $75.1 \%$ male) were included in the analysis; subgroups included: monotherapy $(22.8 \% ; n=115)$, combination therapy $(77.2 \% ; n=390)$. Monotherapy patients were significantly older $(67.7$ vs. 63.4 years; $P=0.0006)$, received ramucirumab approximately 3 months later after diagnosis $(16.9$ vs. 14.1 months; $P$ $=0.0318)$ and more frequently initiated ramucirumab in the third or later lines of treatment $(38.3 \mathrm{vs.} 8.2 \% ; P<0.0001)$ than patients receiving combination therapy. Median overall survival (OS) for monotherapy and combination therapy from the start of second-line therapy was 5.5 months (confidence interval [CI] 4.3, 7.8) and 7.4 months (CI 6.6, 8.8), respectively.

Conclusions The results showed that patients who received ramucirumab monotherapy started ramucirumab therapy later after diagnosis and were older than those who received ramucirumab in combination. Additionally, survival data suggest that outcomes observed in community oncology practices are similar to data from phase 3 clinical trials.
\end{abstract}

Keywords Gastric cancer · Gastroesophageal junction cancer · Ramucirumab · Outcomes research · Overall survival

\section{Introduction}

In the United States (US), approximately 28,000 patients are diagnosed with gastric or gastroesophageal junction (GEJ) cancer annually and 10,960 deaths were estimated in 2017 [1]. The majority of these cases ( 90\%) present with adenocarcinoma, which originates from the mucosa of the stomach [2]. While mortality rates have declined, gastric

Kathleen M. Aguilar

kat.aguilar@mckesson.com

1 Texas Oncology-Baylor Charles A. Sammons Cancer Center, Dallas, TX, USA

2 Eli Lilly and Company, Indianapolis, IN, USA

3 McKesson Specialty Health, 10101 Woodloch Forest Drive, The Woodlands, TX 77380, USA cancer is the second leading cause of global cancer-related death after lung cancer [2]. The prognosis for this disease is poor, with an overall estimated 5-year survival of about 30\% in the US [3]. Although patients with localized disease have a reasonable prognosis through surgical intervention and perioperative treatment, over $60 \%$ of patients are diagnosed at an advanced stage, with an estimated 5-year survival rate of only $5 \%$ for patients with distant metastases.

Chemotherapy and targeted treatments are recommended for patients with advanced or metastatic gastric or GEJ cancer [4]. Systemic, multi-agent chemotherapy is associated with improved survival in the first-line setting [5]. Preferred second-line treatment options recommended by the National Comprehensive Care Network (NCCN) include cytotoxic agents (i.e., docetaxel, paclitaxel, irinotecan, fluorouracil/ irinotecan), as well as the anti-angiogenic, ramucirumab (used alone or in combination with paclitaxel) [4]. The 
introduction of targeted therapies, such as ramucirumab, has provided patients with advanced or metastatic gastric or GEJ cancer with additional treatment options, yet there is uncertainty in routine clinical practice about the outcomes of these therapies outside of a clinical trial setting. Additional evidence would benefit patients and healthcare providers navigate this emerging and complex treatment landscape and identify the most appropriate course of therapy.

Ramucirumab is a human IgG1 monoclonal antibody vascular endothelial growth factor receptor (VEGFR) 2 antagonist [6]. Through blocking activation of VEGFR-2 by VEGF-A and the other VEGF ligands, ramucirumab inhibits the angiogenesis pathways involved in the development and progression of gastric cancer [7]. The safety and efficacy of ramucirumab were demonstrated in two Phase 3 multicenter, international, randomized, double-blind, placebo-controlled trials of patients with advanced or metastatic gastric or GEJ adenocarcinoma who experienced disease progression on or following fluoropyrimidine- or platinum-containing chemotherapy $[8,9]$. In the REGARD trial, ramucirumab monotherapy was associated with significantly improved overall survival (OS) compared to best supportive care (5.2 vs. 3.8 months; hazard ratio [HR] 0.776 [95\% CI 0.603-0.998]; $P=0.047$ ) [8]. Similarly, patients randomized to ramucirumab and paclitaxel in the RAINBOW trial had significantly longer OS compared to those who received paclitaxel alone (9.6 vs. 7.4 months; HR 0.807 [95\% CI 0.678-0.962]; $P=0.017$ ) [9]. In both trials, ramucirumab, either alone or in combination, was generally well tolerated $[8,9]$.

Based on these results, the US Food \& Drug Administration (FDA) approved ramucirumab as a monotherapy (April 21,2014 ) and in combination with paclitaxel (November 5, 2014) for the treatment of advanced or metastatic gastric or GEJ adenocarcinoma with disease progression on or after prior fluoropyrimidine- or platinum-containing chemotherapy $[6,10]$.

The objective of this study was to assess demographic and clinical characteristics as well as treatment patterns and outcomes associated with ramucirumab alone or in combination with cytotoxic agents (e.g., paclitaxel) for the treatment of gastric or GEJ adenocarcinoma. The treatment patterns and survival outcomes among patients who received either ramucirumab monotherapy or in combination were investigated. Additionally, factors associated with monotherapy and combination therapy utilization were explored.

\section{Methods}

\section{Study Design}

This was a retrospective observational cohort study that examined patient profiles, treatment patterns and outcomes for patients with gastric or GEJ cancer treated with ramucirumab who received healthcare services at US Oncology Network (USON) clinics. The date of initiation of a ramucirumab-containing regimen was defined as the index date. Eligible patients were 18 years of age or older at the index date who had a documented diagnosis of gastric or GEJ cancer, initiated ramucirumab between April 21, 2014 and June 30, 2016, received care at USON sites utilizing the full electronic healthcare record (EHR) capacities at the time of treatment and had $\geq 2$ office visits during the study observation period. Patients enrolled in clinical trials at any time during the study period were excluded because they had clinical scenarios that deviated from the population of interest.

The EHR of the USON, iKnowMed (iKM), was used in this study. iKM is an oncology-specific EHR system that captures outpatient practice encounter history for patients who receive care within the USON, including, but not limited to laboratory tests, diagnosis, therapy administration, line of therapy (LOT), cancer stage, comorbidities and performance status. iKM captures data on outpatient medical oncology care for patients treated across the US (19 states). Overall, the iKM EHR system captures data on approximately $10 \%$ of newly diagnosed cancer patients in the US.

To supplement available vital status information in iKM, the Social Security Death Index (SSDI) was used to estimate OS. OS was defined as the interval between the index date and the date of death as documented in the SSDI and iKM EHR database. Patients alive at the end of the database were censored on the study end date or the last visit date available in the database, whichever occurred first. Because the study derived data mainly from the iKM database to meet the objectives, an intent-to-treat perspective was applied.

\section{Statistical analysis}

Patient profiles, treatment patterns and outcomes were assessed for all patients identified in the iKM database who met inclusion criteria, as well as for the subgroups of patients receiving monotherapy or combination therapy. Classification of patients into the monotherapy and combination subgroups was based on their initial ramucirumab regimen. For example, if a patient began ramucirumab monotherapy and advanced to a new LOT with a combination ramucirumab regimen, that patient was considered to be a monotherapy subgroup member.

Demographic, clinical and treatment characteristics were summarized for the entire patient cohort and subgroups. Continuous variables were described by mean, standard deviation, median and interquartile range (IQR). Categorical variables 
Table 1 Baseline and clinical characters characteristics of gastric or GEJ cancer patients receiving ramucirumab

\begin{tabular}{|c|c|c|c|c|}
\hline Analysis variable & $\begin{array}{l}\text { Total study cohort }(N= \\
505)\end{array}$ & $\begin{array}{l}\text { Monotherapy sub- } \\
\text { group }(n=115)\end{array}$ & $\begin{array}{l}\text { Combination } \\
\text { therapy subgroup }(n \\
=390)\end{array}$ & $P$ value \\
\hline Monotherapy use at index, $n(\%)$ & $115(22.77 \%)$ & & & \\
\hline Combination therapy use at index, $n(\%)$ & $390(77.23 \%)$ & & & \\
\hline Age at index (years) & & & & $0.0006^{\mathrm{d}}$ \\
\hline Mean (SD) & $64.41(11.42)$ & $67.74(11.63)$ & $63.42(11.19)$ & \\
\hline Median (IQR) & $65.06(57.39,72.31)$ & $67.50(60.42,75.81)$ & $64.11(56.97,70.95)$ & \\
\hline Gender, $n(\%)$ & & & & $0.7486^{\mathrm{e}}$ \\
\hline Female & $126(24.95 \%)$ & $30(26.09 \%)$ & $96(24.62 \%)$ & \\
\hline Male & $379(75.05 \%)$ & $85(73.91 \%)$ & $294(75.39 \%)$ & \\
\hline Race, $n(\%)$ & & & & $0.9558^{\mathrm{e}}$ \\
\hline Black & $32(6.34 \%)$ & $8(6.96 \%)$ & $24(6.15 \%)$ & \\
\hline White & $407(80.59 \%)$ & $93(80.87 \%)$ & $314(80.51 \%)$ & \\
\hline Other & $25(4.95 \%)$ & $6(5.22 \%)$ & $19(4.87 \%)$ & \\
\hline Not documented & $41(8.12 \%)$ & $8(6.96 \%)$ & $33(8.46 \%)$ & \\
\hline Ethnicity, $n(\%)$ & & & & $0.2817^{\mathrm{e}}$ \\
\hline Hispanic or Latino & $75(14.85 \%)$ & $13(11.30 \%)$ & $62(15.90 \%)$ & \\
\hline Not Hispanic or Latino & $392(77.62 \%)$ & $90(78.26 \%)$ & $302(77.44 \%)$ & \\
\hline Not documented & $38(7.53 \%)$ & $12(10.44 \%)$ & $26(6.67 \%)$ & \\
\hline Geographic region, $n(\%)$ & & & & $0.0037^{\mathrm{e}}$ \\
\hline Midwest & $64(12.67 \%)$ & $19(16.52 \%)$ & $45(11.54 \%)$ & \\
\hline Northeast & $19(3.76 \%)$ & $7(6.09 \%)$ & $12(3.08 \%)$ & \\
\hline South & $289(57.23 \%)$ & $49(42.61 \%)$ & $240(61.54 \%)$ & \\
\hline West & $133(26.34 \%)$ & $40(34.78 \%)$ & $93(23.85 \%)$ & \\
\hline Payer information, $n(\%)$ & & & & $0.0220^{\mathrm{c}}$ \\
\hline Medicaid & $22(4.36 \%)$ & $3(2.61 \%)$ & $19(4.87 \%)$ & \\
\hline Medicare & $197(39.01 \%)$ & $54(46.96 \%)$ & $143(36.67 \%)$ & \\
\hline Private & $174(34.46 \%)$ & $30(26.09 \%)$ & $144(36.92 \%)$ & \\
\hline Other & $18(3.56 \%)$ & $1(0.87 \%)$ & $17(4.36 \%)$ & \\
\hline Not documented & $94(18.61 \%)$ & $27(23.48 \%)$ & $67(17.18 \%)$ & \\
\hline Physician specialty, $n(\%)$ & & & & $0.7943^{c}$ \\
\hline Hematology and medical oncology & $450(89.11 \%)$ & $104(90.44 \%)$ & $346(88.72 \%)$ & \\
\hline Internal medicine & $1(0.20 \%)$ & $0(0.00 \%)$ & $1(0.26 \%)$ & \\
\hline Not documented & $54(10.69 \%)$ & $11(9.57 \%)$ & $43(11.03 \%)$ & \\
\hline Physician patient volume, $n(\%)$ & & & & $0.0205^{\mathrm{c}}$ \\
\hline$<100$ patients/year & $374(74.06 \%)$ & $96(83.48 \%)$ & $278(71.28 \%)$ & \\
\hline 100-199 patients/year & $121(23.96 \%)$ & $17(14.78 \%)$ & $104(26.67 \%)$ & \\
\hline $200+$ patients/year & $10(1.98 \%)$ & $2(1.74 \%)$ & $8(2.05 \%)$ & \\
\hline BMI category at baseline, $n(\%)$ & & & & $0.6063^{\mathrm{c}}$ \\
\hline Normal & $216(42.77 \%)$ & $53(46.09 \%)$ & $163(41.80 \%)$ & \\
\hline Obese & $95(18.81 \%)$ & $18(15.65 \%)$ & $77(19.74 \%)$ & \\
\hline Overweight & $114(22.57 \%)$ & $27(23.48 \%)$ & $87(22.31 \%)$ & \\
\hline Underweight & $74(14.65 \%)$ & $14(12.17 \%)$ & $60(15.39 \%)$ & \\
\hline Not documented & $6(1.19 \%)$ & $3(2.61 \%)$ & $3(0.77 \%)$ & \\
\hline Smoking status at baseline, $n(\%)$ & & & & $0.4618^{c}$ \\
\hline Current & $76(15.05 \%)$ & $15(13.04 \%)$ & $61(15.64 \%)$ & \\
\hline Former & $238(47.13 \%)$ & $60(52.17 \%)$ & $178(45.64 \%)$ & \\
\hline Never & $186(36.83 \%)$ & $39(33.91 \%)$ & $147(37.69 \%)$ & \\
\hline Not documented & $5(0.99 \%)$ & $1(0.87 \%)$ & $4(1.03 \%)$ & \\
\hline Histological subtype, $n(\%)$ & & & & $0.4091^{\mathrm{c}}$ \\
\hline
\end{tabular}


Table 1 (continued)

Analysis variable
Total study cohort $(N=$ 505)
Monotherapy subgroup $(n=115)$
Combination therapy subgroup $(n$ $=390$ )

\section{Adenocarcinoma}

Signet ring cell carcinoma

Not documented

Time from initial gastric or GEJ cancer diagnosis to start of ramucirumab (m)

Patients with available data

Mean (SD)

Median (IQR)

Time since prior therapy to start of ramucirumab (m)

Patients with available data

Mean (SD)

Median (IQR)

Primary tumor locations, $n(\%)$

Greater curvature

Lesser curvature of stomach, unspecified

Cardio-esophageal junction

Antrum of stomach NOS

Lower thoracic esophagus

Body of stomach

Lower third of esophagus

Gastroesophageal junction

Other

Not documented

Stage at initial gastric or GEJ cancer diagnosis, $n(\%)$

I

II

III

IV

Not documented

Prior cancer diagnosis, $n(\%)$

No

Yes

Prior cancer diagnosis location (if applicable), $n(\%)$

Pancreas

Prostate

Cancer, unknown primary

Breast cancer, female

Other

Use of ramucirumab before/after November 2014 combination therapy approval, $n(\%)$

Monotherapy prior to November 2014 FDA approval

Combination therapy prior to November 2014 FDA approval

Monotherapy after November 2014 FDA approval

Combination therapy after November 2014 FDA approval

Number of agents used in line of therapy prior to ramucirumab (excluding mesna and leucovorin)

Patients with available data

$30(5.94 \%)$
$4(0.79 \%)$
$471(93.27 \%)$

503

$14.70(14.69)$

$10.09(5.78,18.13)$

433

$2.07(2.62)$

$0.99(0.69,2.33)$

$11(2.18 \%)$

$13(2.57 \%)$

$14(2.77 \%)$

$23(4.55 \%)$

$25(4.95 \%)$

$26(5.15 \%)$

$46(9.11 \%)$

$93(18.42 \%)$

$41(8.12 \%)$

$213(42.18 \%)$

$19(3.76 \%)$

$72(14.26 \%)$

$99(19.60 \%)$

$285(56.44 \%)$

$30(5.94 \%)$

$451(89.31 \%)$

$54(10.69 \%)$

$5(0.99 \%)$

$5(0.99 \%)$

$8(1.58 \%)$

$9(1.78 \%)$

$31(6.14 \%)$

$71(53.79 \%)$

$61(46.21 \%)$

$44(11.80 \%)$

$329(88.20 \%)$

$\begin{array}{ll}3(2.61 \%) & 27(6.92 \%) \\ 1(0.87 \%) & 3(0.77 \%) \\ 111(96.52 \%) & 360(92.31 \%)\end{array}$

$0.0318^{\mathrm{d}}$

$115 \quad 388$

$16.86(15.81) \quad 14.06(14.29)$

$12.55(6.57,22.73) \quad 9.69(5.50,17.56)$

$0.1151^{\mathrm{d}}$

$90 \quad 343$

$1.42(1.22) \quad 2.24(2.86)$

$0.92(0.69,1.71)$

$0.99(0.69,2.63)$

$0.5943^{\mathrm{f}}$

$5(4.35 \%) \quad 6(1.54 \%)$

$3(2.61 \%) \quad 10(2.56 \%)$

$3(2.61 \%) \quad 11(2.82 \%)$

$5(4.35 \%) \quad 18(4.62 \%)$

$4(3.48 \%) \quad 21(5.38 \%)$

$6(5.22 \%) \quad 20(5.13 \%)$

$6(5.22 \%) \quad 40(10.26 \%)$

$19(16.52 \%) \quad 74(18.97 \%)$

$10(8.70 \%) \quad 31(7.95 \%)$

$54(46.96 \%) \quad 159(40.77 \%)$

$0.9717^{\mathrm{c}}$

$4(3.48 \%) \quad 15(3.85 \%)$

$16(13.91 \%) \quad 56(14.36 \%)$

$24(20.87 \%) \quad 75(19.23 \%)$

$62(53.91 \%) \quad 223(57.18 \%)$

$9(7.83 \%) \quad 21(5.39 \%)$

$0.5587^{\mathrm{e}}$

$101(87.83 \%) \quad 350(89.74 \%)$

$14(12.17 \%) \quad 40(10.26 \%)$

$1(0.87 \%)$

$4(1.03 \%)$

$1.000^{\mathrm{c}}$

$0(0.00 \%)$

$5(1.28 \%)$

$0.2224^{\mathrm{c}}$

$2(1.74 \%)$

$6(1.54 \%)$

$1.000^{\mathrm{c}}$

$4(3.48 \%)$

$5(1.28 \%)$

$0.1247^{\mathrm{c}}$

$7(6.09 \%)$ 
Table 1 (continued)

\begin{tabular}{|c|c|c|c|c|}
\hline Analysis variable & $\begin{array}{l}\text { Total study cohort }(N= \\
505)\end{array}$ & $\begin{array}{l}\text { Monotherapy sub- } \\
\text { group }(n=115)\end{array}$ & $\begin{array}{l}\text { Combination } \\
\text { therapy subgroup ( } n \\
=390)\end{array}$ & $P$ value \\
\hline Mean (SD) & $2.38(0.79)$ & $2.01(0.79)$ & $2.47(0.76)$ & \\
\hline Median (IQR) & $2(2,3)$ & $2(1,3)$ & $3(2,3)$ & \\
\hline Number of prior LOTs, $n(\%)$ & & & & $0.2155^{\mathrm{c}}$ \\
\hline 1 & $338(66.93 \%)$ & $75(65.22 \%)$ & $263(67.44 \%)$ & \\
\hline 2 & $79(15.64 \%)$ & $11(9.57 \%)$ & $68(17.44 \%)$ & \\
\hline $3+$ & $15(2.97 \%)$ & $4(3.48 \%)$ & $11(2.82 \%)$ & \\
\hline No prior treatment documented in $\mathrm{EHR}^{\mathrm{a}}$ & $72(14.26 \%)$ & $25(21.74 \%)$ & $47(12.05 \%)$ & \\
\hline Number of prior LOTs undefined ${ }^{b}$ & $1(0.20 \%)$ & $0(0.00 \%)$ & $1(0.26 \%)$ & \\
\hline $\begin{array}{l}\text { Prior treatments for gastric or GEJ cancer prior to ramu- } \\
\text { cirumab initiation, } n(\%)\end{array}$ & $433(85.74 \%)$ & $90(78.26 \%)$ & $343(87.95 \%)$ & $0.0090^{\mathrm{e}}$ \\
\hline Anti-angiogenic-containing & $1(0.20 \%)$ & $0(0.00 \%)$ & $1(0.26 \%)$ & $1.000^{\mathrm{c}}$ \\
\hline Antineoplastic-containing & $6(1.19 \%)$ & $0(0.00 \%)$ & $6(1.54 \%)$ & $0.1809^{c}$ \\
\hline Irinotecan-containing & $48(9.51 \%)$ & $17(14.78 \%)$ & $31(7.95 \%)$ & $0.0281^{\mathrm{e}}$ \\
\hline Anthracycline-containing & $67(13.27 \%)$ & $8(6.96 \%)$ & $59(15.13 \%)$ & $0.0232^{\mathrm{e}}$ \\
\hline Trastuzumab-containing & $75(14.85 \%)$ & $16(13.91 \%)$ & $59(15.13 \%)$ & $0.7474^{\mathrm{e}}$ \\
\hline Taxane-containing & $146(28.91 \%)$ & $38(33.04 \%)$ & $108(27.69 \%)$ & $0.2660^{\mathrm{e}}$ \\
\hline Platinum-containing & $316(62.57 \%)$ & $47(40.87 \%)$ & $269(68.97 \%)$ & $<0.0001^{\mathrm{e}}$ \\
\hline Fluoropyrimidine-containing & $334(66.14 \%)$ & $49(42.61 \%)$ & $285(73.08 \%)$ & $<0.0001^{\mathrm{e}}$ \\
\hline Evidence of metastatic disease at baseline, $n(\%)$ & & & & $0.5435^{\mathrm{c}}$ \\
\hline No & $16(3.17 \%)$ & $2(1.74 \%)$ & $14(3.59 \%)$ & \\
\hline Yes & $489(96.83 \%)$ & $113(98.26 \%)$ & $376(96.41 \%)$ & \\
\hline \multicolumn{5}{|l|}{ Location of metastases (if applicable), $n(\%)$} \\
\hline Brain & $14(2.77 \%)$ & $7(6.09 \%)$ & $7(1.80 \%)$ & $0.0138^{\mathrm{e}}$ \\
\hline Bone & $60(11.88 \%)$ & $16(13.91 \%)$ & $44(11.28 \%)$ & $0.4435^{\mathrm{e}}$ \\
\hline Lung & $63(12.48 \%)$ & $12(10.44 \%)$ & $51(13.08 \%)$ & $0.4511^{\mathrm{e}}$ \\
\hline Peritoneum & $96(19.01 \%)$ & $21(18.26 \%)$ & $75(19.23 \%)$ & $0.8158^{\mathrm{e}}$ \\
\hline Liver & $152(30.10 \%)$ & $38(33.04 \%)$ & $114(29.23 \%)$ & $0.4334^{\mathrm{e}}$ \\
\hline Other & $110(21.78 \%)$ & $17(14.78 \%)$ & $93(23.85 \%)$ & $0.0385^{\mathrm{e}}$ \\
\hline Not documented & $289(57.23 \%)$ & $67(58.26 \%)$ & $222(56.92 \%)$ & \\
\hline $\begin{array}{l}\text { ECOG performance score at initiation of ramucirumab } \\
\text { use, } n(\%)\end{array}$ & & & & $0.4136^{\mathrm{c}}$ \\
\hline 0 & $25(4.95 \%)$ & $3(2.61 \%)$ & $22(5.64 \%)$ & \\
\hline 1 & $182(36.04 \%)$ & $41(35.65 \%)$ & $141(36.15 \%)$ & \\
\hline $2+$ & $51(10.10 \%)$ & $13(11.30 \%)$ & $38(9.74 \%)$ & \\
\hline Not documented & $247(48.91 \%)$ & $58(50.44 \%)$ & $189(48.46 \%)$ & \\
\hline $\begin{array}{l}\text { Weight loss (3 months prior to initiation of ramucirumab), } \\
n(\%)\end{array}$ & & & & $0.5878^{\mathrm{c}}$ \\
\hline$<10 \%$ & $124(24.55 \%)$ & $31(26.96 \%)$ & $93(23.85 \%)$ & \\
\hline$\geq 10 \%$ & $10(1.98 \%)$ & $1(0.87 \%)$ & $9(2.31 \%)$ & \\
\hline No weight loss & $371(73.47 \%)$ & $83(72.17 \%)$ & $288(73.85 \%)$ & \\
\hline \multicolumn{5}{|l|}{ Comorbidities at initiation of ramucirumab, $n(\%)$} \\
\hline Liver & $5(0.99 \%)$ & $1(0.87 \%)$ & $4(1.03 \%)$ & $1.000^{\mathrm{c}}$ \\
\hline Renal & $9(1.78 \%)$ & $3(2.61 \%)$ & $6(1.54 \%)$ & $0.4323^{\mathrm{c}}$ \\
\hline Diabetes & $21(4.16 \%)$ & $1(0.87 \%)$ & $20(5.13 \%)$ & $0.0587^{\mathrm{c}}$ \\
\hline Pulmonary & $36(7.13 \%)$ & $6(5.22 \%)$ & $30(7.69 \%)$ & $0.3647^{\mathrm{e}}$ \\
\hline Neuropathy & $43(8.52 \%)$ & $6(5.22 \%)$ & $37(9.49 \%)$ & $0.1494^{\mathrm{e}}$ \\
\hline Cardiovascular & $45(8.91 \%)$ & $7(6.09 \%)$ & $38(9.74 \%)$ & $0.2265^{\mathrm{e}}$ \\
\hline Hematological & $120(23.76 \%)$ & $34(29.57 \%)$ & $86(22.05 \%)$ & $0.0962^{\mathrm{e}}$ \\
\hline Other & $223(44.16 \%)$ & $43(37.39 \%)$ & $180(46.15 \%)$ & $0.0963^{\mathrm{e}}$ \\
\hline
\end{tabular}


Table 1 (continued)

\begin{tabular}{|c|c|c|c|c|}
\hline Analysis variable & $\begin{array}{l}\text { Total study cohort }(N= \\
505)\end{array}$ & $\begin{array}{l}\text { Monotherapy sub- } \\
\text { group }(n=115)\end{array}$ & $\begin{array}{l}\text { Combination } \\
\text { therapy subgroup }(n \\
=390)\end{array}$ & $P$ value \\
\hline \multicolumn{5}{|c|}{$\begin{array}{l}\text { Toxicity/symptoms at initiation of ramucirumab (60-day } \\
\text { period before index event), } n(\%)\end{array}$} \\
\hline Neutropenia & $22(4.36 \%)$ & $8(6.96 \%)$ & $14(3.59 \%)$ & $0.1201^{\mathrm{e}}$ \\
\hline Edema & $24(4.75 \%)$ & $7(6.09 \%)$ & $17(4.36 \%)$ & $0.4440^{\mathrm{e}}$ \\
\hline Abdominal pain/bloating & $24(4.75 \%)$ & $2(1.74 \%)$ & $22(5.64 \%)$ & $0.1306^{\mathrm{c}}$ \\
\hline Neuropathy & $25(4.95 \%)$ & $7(6.09 \%)$ & $18(4.62 \%)$ & $0.5226^{\mathrm{e}}$ \\
\hline Diarrhea & $28(5.55 \%)$ & $7(6.09 \%)$ & $21(5.39 \%)$ & $0.7724^{\mathrm{e}}$ \\
\hline Nausea & $102(20.20 \%)$ & $13(11.30 \%)$ & $89(22.82 \%)$ & $0.0069^{\mathrm{e}}$ \\
\hline Other & $46(9.11 \%)$ & $9(7.83 \%)$ & $37(9.49 \%)$ & $0.5864^{\mathrm{e}}$ \\
\hline
\end{tabular}

${ }^{a}$ These patients may have prior treatment outside of the US Oncology Network (USON)

${ }^{\mathrm{b}}$ This patient had evidence of prior treatment within the USON but the number of LOTs was not defined in the EHR database

${ }^{\mathrm{c}} P$ value calculated based on a Fisher's Exact test

${ }^{\mathrm{d}} P$ value calculated based on a Kruskal-Wallis test

${ }^{\mathrm{e}} P$ value calculated based on a $\chi^{2}$ test

${ }^{\mathrm{f}}$ Monte Carlo estimate

were defined by patient count and percentage. To make statistical comparisons between the subgroups, Pearson $\chi^{2}$ or Fisher's exact test were used to analyze categorical variables and Kruskal-Wallis tests were conducted for continuous variables.

An alpha level of 0.05 was the primary criterion for statistical significance of this study. Results were reported in aggregate using SAS ${ }^{\circledR} 9.4$ (SAS Institute Inc., Cary, NC, US).

Stepwise multivariable logistic regression models were used to evaluate predictors for the use of ramucirumab monotherapy versus combination therapy. Predictors, consisting of baseline demographic and clinical factors, found to be significant at the 0.25 level were entered into the model, while final predictors retained in the model had to be significant at the 0.10 level. To increase statistical power, predictors identified in the final model were refitted in a logistic regression model to avoid exclusion of patients with missing data due to unselected predictors.

Kaplan-Meier curves were constructed for the monotherapy and combination therapy subgroups to illustrate OS and time to treatment discontinuation (TTTD) profiles and medians with their respective $95 \%$ confidence intervals (CIs). Survival rates were calculated at 6, 9, 12 and 18 months for OS. Conversely, treatment discontinuation rates for TTTD were calculated at 3-, 6-, 9- and 12-month periods.

Similar to evaluating baseline and clinical predictive factors in the multivariable logistic regression models, the same was done for univariate and multivariable Cox proportional hazards regression analyses for OS and TTTD. That is, stepwise multivariable Cox proportional hazards regression models utilized a 0.25 significance level for predictors to be entered into the model and a 0.10 level for them to remain in the model.
Demographic and clinical confounding factors were included in the multivariable logistic regression and Cox proportional hazards regression models based on their clinical relevance and/or the univariate significance level.

\section{Results}

\section{Patient demographic and clinical characteristics}

A total of 505 patients (mean age 64.4 years; $75.1 \%$ male; $80.6 \%$ White; $57.2 \%$ treated in Southern US; Table 1) were included in the analysis; subgroups included: monotherapy $(22.8 \% ; n=115)$ and combination therapy $(77.2 \% ; n=390$; Fig. 1). The majority of the study population, $73.5 \%$, had either Medicare $(39.0 \%)$ or private $(34.5 \%)$ insurance (Table 1). Healthcare services received in hematological and medical oncology settings were the primary source for this study population (89.1\%). Physicians treating less than 100 patients annually represented almost three-fourths of the care administered (74.1\%). Of the overall study population, $42.8 \%$ had normal weight, while $22.6 \%$ and $18.8 \%$ were overweight and obese, respectively. Histology was infrequently documented, with a record of adenocarcinoma for $5.9 \%$ of patients, signet ring carcinoma for $0.8 \%$ of patients and missing information for $93.3 \%$. Compared to those who received combination therapy, the monotherapy subgroup was significantly older (67.7 vs. 63.4 years; $P=0.0006$ ).

Patients had been diagnosed with gastric or GEJ cancer for an average of 14.7 months $( \pm 14.7)$ at the index date, with a mean of 2.1 months $( \pm 2.6)$ between their prior therapy and the start of ramucirumab treatment (Table 1). Most 
Patients with a documented diagnosis of gastric or esophageal cancer (including GEJ)

$\mathrm{N}=\mathbf{2 1 , 5 6 3}$

Patients seen at USON clinics utilizing the full EHR capacities of iKM

$\mathrm{n}=\mathbf{1 9 , 1 5 0}$

Patient with $\geq 2$ office visits at USON clinics (second visit after the index date)

$\mathrm{n}=16,751$

Exclusion of patients enrolled in a clinical trial during the study period.

$n=16,637$

Patients who received an order for ramucirumab between 4/21/14-6/30/16 and had evidence of the order being administered $\mathrm{N}=505$

Patients aged $\geq 18$ years at the index date (initiation of ramucirumab)

$\mathrm{N}=505$

\section{Subgroups}

Monotherapy
subgroup count
$\mathbf{n = 1 1 5}$$\quad \begin{gathered}\text { Combination therapy } \\ \text { subgroup count } \\ \mathbf{n = 3 9 0}\end{gathered}$

Fig. 1 CONSORT Diagram

patients $(56.4 \%)$ were diagnosed with Stage IV disease and did not have a prior cancer diagnosis documented in the EHR (89.3\%). Prior to starting treatment with ramucirumab, approximately $85 \%$ of patients had received prior lines of therapy. On average, patients received $2.4( \pm 0.8)$ agents in the LOT prior to the ramucirumab-containing regimen (median 2; IQR 2, 3).

Compared to patients in the combination therapy subgroup, the duration between diagnosis and ramucirumab initiation was approximately 3 months longer for those who receivedmonotherapy (mean 16.9 [ \pm 15.8 ] vs. 14.1 [14.3] months; $P=0.0318$; Table 1 ). A significantly higher number of patients who received ramucirumab in combination received prior therapy compared to the monotherapy subgroup ( 88.0 vs. $78.3 \%$ with prior therapies; $P=0.009$ ). Furthermore, a difference of prior regimens was observed between the treatment groups. A higher use of irinotecan-, anthracycline-, platinum- and fluoropyrimidine-containing regimens were observed in the combination subgroup compared to patients receiving monotherapy $(P=0.0281$, $P=0.0232, P<0.0001$ and $P<0.0001$, respectively).

\section{Treatment patterns}

The majority $(80.6 \%)$ of patients began ramucirumab treatment in the second-line setting (Table 2). Approximately $70 \%$ of patients did not receive a subsequent treatment following discontinuation of ramucirumab. Ramucirumab dose modifications occurred among 14 (2.8\%) patients; 12 (3.1\%) who received combination therapy and $2(1.7 \%)$ who received monotherapy. A significantly higher proportion of monotherapy patients initiated ramucirumab in the third-line setting or beyond, than those who received ramucirumab in combination (38.3 vs. $8.2 \%$, respectively; $P<0.0001$ ). While most patients $(53.8 \%)$ received monotherapy following the approval of ramucirumab as a single agent (April 2014-November 2014), only $11.8 \%$ of patients received monotherapy after the FDA approval of ramucirumab plus paclitaxel in November 2014 (Table 1). Throughout the study period, 5 patients switched from ramucirumab monotherapy to combination therapy, while 4 switched from combination therapy to monotherapy.

\section{Clinical outcomes}

The mean overall follow-up time from the index diagnosis to the last contact date, end of study period or date of death, whichever came first, was 11.1 months ( \pm 6.4 ; Table 2 ). The mean duration of follow-up was significantly greater among patients who received combination therapy compared to those who received monotherapy $(11.8[ \pm 6.3]$ vs. 8.8 [ \pm 6.3] months; $P<0.0001)$.

Among patients who received ramucirumab in the second-line setting, the median OS durations for the monotherapy and combination therapy subgroups were 5.5 months (CI $4.3,7.8$ ) and 7.4 months (CI 6.6, 8.8), respectively (Fig. 2). The multivariable Cox proportional hazard model identified several predictors of OS for patients who received ramucirumab monotherapy (Table 3). Hispanic or Latino ethnicity, location of clinic (Northeast vs. South) and diarrhea were associated with a decreased risk of death. In contrast, patients with liver or peritoneal metastases had an increased risk of death than those without.

Among patients who received combination therapy, Hispanic or Latino ethnicity and body mass index (BMI; obese vs. normal) appeared to have a protective effect on OS (Table 3). Patients with an Eastern Cooperative Oncology Group (ECOG) performance status score of 2 or greater, hematological and pulmonary comorbidities, lung 
Table 2 Treatment patterns of gastric or GEJ cancer patients receiving ramucirumab

\begin{tabular}{|c|c|c|c|c|}
\hline Analysis variable & Total study cohort $(N=505)$ & $\begin{array}{l}\text { Monotherapy sub- } \\
\text { group }(n=115)\end{array}$ & $\begin{array}{l}\text { Combination therapy } \\
\text { subgroup }(n=390)\end{array}$ & $P$ value \\
\hline \multicolumn{5}{|l|}{ Regimen used for index, $n(\%)$} \\
\hline Ramucirumab monotherapy & $115(22.77 \%)$ & & & \\
\hline Ramucirumab + paclitaxel & $381(75.45 \%)$ & & & \\
\hline Ramucirumab + other combination agents & $9(1.78 \%)$ & & & \\
\hline \multicolumn{5}{|l|}{ Switched ramucirumab regimen, $n(\%)$} \\
\hline Combination to monotherapy & $4(44.44 \%)$ & & & \\
\hline Monotherapy to combination & $5(55.56 \%)$ & & & \\
\hline First LOT with ramucirumab, $n(\%)$ & & & & $<0.0001^{\mathrm{a}}$ \\
\hline LOT1 & $15(2.97 \%)$ & $4(3.48 \%)$ & $11(2.82 \%)$ & \\
\hline LOT2 & $407(80.59 \%)$ & $65(56.52 \%)$ & $342(87.69 \%)$ & \\
\hline LOT3 & $58(11.49 \%)$ & $31(26.96 \%)$ & $27(6.92 \%)$ & \\
\hline LOT4+ & $18(3.56 \%)$ & $13(11.30 \%)$ & $5(1.28 \%)$ & \\
\hline LOT unknown & $7(1.39 \%)$ & $2(1.74 \%)$ & $5(1.28 \%)$ & \\
\hline Duration of follow-up (months) & & & & $<0.0001^{\mathrm{b}}$ \\
\hline Mean (SD) & $11.13(6.43)$ & $8.77(6.28)$ & $11.83(6.32)$ & \\
\hline Median (IQR) & $9.86(6.28,15.19)$ & $6.90(3.78,12.23)$ & $10.64(7.00,15.68)$ & \\
\hline \multicolumn{5}{|l|}{$\begin{array}{l}\text { Treatment immediately following ramucirumab } \\
\text { discontinuation, } n(\%)\end{array}$} \\
\hline Subsequent treatment & $145(28.71 \%)$ & $35(30.44 \%)$ & $110(28.21 \%)$ & $0.6423^{\mathrm{c}}$ \\
\hline Anthracycline-containing & $6(1.19 \%)$ & $1(0.87 \%)$ & $5(1.28 \%)$ & $1.000^{\mathrm{a}}$ \\
\hline Antineoplastic-containing & $9(1.78 \%)$ & $4(3.48 \%)$ & $5(1.28 \%)$ & $0.1247^{\mathrm{a}}$ \\
\hline Trastuzumab-containing & $17(3.37 \%)$ & $7(6.09 \%)$ & $10(2.56 \%)$ & $0.0657^{\mathrm{c}}$ \\
\hline Platinum-containing & $23(4.55 \%)$ & $8(6.96 \%)$ & $15(3.85 \%)$ & $0.1598^{\mathrm{c}}$ \\
\hline Taxane-containing & $23(4.55 \%)$ & $7(6.09 \%)$ & $16(4.10 \%)$ & $0.3697^{\mathrm{c}}$ \\
\hline Irinotecan-containing & $41(8.12 \%)$ & $6(5.22 \%)$ & $35(8.97 \%)$ & $0.1949^{c}$ \\
\hline Fluoropyrimidine-containing & $51(10.10 \%)$ & $13(11.30 \%)$ & $38(9.74 \%)$ & $0.6254^{\mathrm{c}}$ \\
\hline Duration of ramucirumab therapy (months) ${ }^{\mathrm{d}}$ & & & & $0.2378^{\mathrm{b}}$ \\
\hline Mean (SD) & $3.13(3.23)$ & $2.91(3.31)$ & $3.20(3.21)$ & \\
\hline Median (IQR) & $2.10(0.95,4.24)$ & $1.87(0.95,4.24)$ & $2.30(0.95,4.6)$ & \\
\hline Number of ramucirumab infusions across all LOTs & & & & $0.6329^{b}$ \\
\hline Patients with available data & 511 & 117 & 394 & \\
\hline Number of ramucirumab infusions & 3507 & 791 & 2716 & \\
\hline Mean (SD) & $6.86(6.11)$ & $6.76(6.69)$ & $6.89(5.94)$ & \\
\hline Median (IQR) & $5(3,9)$ & $5(3,7)$ & $5(3,10)$ & \\
\hline LOT1 & & & & $0.5109^{b}$ \\
\hline Patients with available data ${ }^{\mathrm{f}}$ & 15 & 4 & 11 & \\
\hline Number of ramucirumab infusions & 83 & 22 & 61 & \\
\hline Mean (SD) & $5.53(4.19)$ & $5.50(2.08)$ & $5.55(4.82)$ & \\
\hline Median (IQR) & $5(3,8)$ & $5.5(4,7)$ & $4(2,8)$ & \\
\hline LOT2 & & & & $0.4358^{\mathrm{b}}$ \\
\hline Patients with available data ${ }^{\mathrm{f}}$ & 408 & 65 & 343 & \\
\hline Number of ramucirumab infusions & 2898 & 500 & 2398 & \\
\hline Mean (SD) & $7.10(6.33)$ & $7.69(7.62)$ & $6.99(6.07)$ & \\
\hline Median (IQR) & $5(3,10)$ & $5(4,8)$ & $5(3,10)$ & \\
\hline LOT3 & & & & $0.6567^{\mathrm{b}}$ \\
\hline Patients with available data ${ }^{\mathrm{f}}$ & 61 & 33 & 28 & \\
\hline Number of ramucirumab infusions & 366 & 200 & 166 & \\
\hline Mean (SD) & $6.00(4.98)$ & $6.06(4.66)$ & $5.93(5.42)$ & \\
\hline Median (IQR) & $5(3,6)$ & $5(3,6)$ & $4.5(2.5,6.5)$ & \\
\hline
\end{tabular}


Table 2 (continued)

\begin{tabular}{|c|c|c|c|c|}
\hline Analysis variable & Total study cohort $(N=505)$ & $\begin{array}{l}\text { Monotherapy sub- } \\
\text { group }(n=115)\end{array}$ & $\begin{array}{l}\text { Combination therapy } \\
\text { subgroup }(n=390)\end{array}$ & $P$ value \\
\hline LOT4+ & & & & $0.0190^{\mathrm{b}}$ \\
\hline Patients with available data ${ }^{\mathrm{f}}$ & 19 & 13 & 6 & \\
\hline Number of ramucirumab infusions & 104 & 64 & 40 & \\
\hline Mean (SD) & $5.47(6.08)$ & $4.92(7.10)$ & $6.67(3.08)$ & \\
\hline Median (IQR) & $4(2,7)$ & $2(2,4)$ & $6(4,8)$ & \\
\hline LOT Unknown & & & & $0.0651^{\mathrm{b}}$ \\
\hline Patients with available data ${ }^{\mathrm{f}}$ & 8 & 2 & 6 & \\
\hline Number of ramucirumab infusions & 56 & 5 & 51 & \\
\hline Mean (SD) & $7(5.29)$ & $2.50(0.71)$ & $8.50(5.32)$ & \\
\hline Median (IQR) & $4.5(3,11.5)$ & $2.5(2,3)$ & $7.5(4,13)$ & \\
\hline Ramucirumab dose modifications (cycles) & & & & $0.2216^{\mathrm{b}}$ \\
\hline Patients with available data & 14 & 2 & 12 & \\
\hline Mean (SD) & $3.29(1.90)$ & $5(2.83)$ & $3.00(1.71)$ & \\
\hline Median (IQR) & $3(2,4)$ & $5(3,7)$ & $3(2,3.5)$ & \\
\hline
\end{tabular}

${ }^{\text {a }} P$ value calculated based on a Fisher's Exact test

${ }^{\mathrm{b}} P$ value calculated based on a Kruskal-Wallis test

${ }^{\mathrm{c}} P$ value calculated based on a $\chi^{2}$ test

${ }^{\mathrm{d}}$ Patients with ongoing treatment were censored for overall follow-up, duration of therapy and survival estimates. In total, 100 patients were censored due to ongoing treatment. Ongoing treatment was defined as a treatment administration between 5 May 2016 and 30 June 2016

ePatients with ongoing treatment were not censored for calculations of the number of dose modifications or infusions

Patients may have received ramucirumab in multiple LOTs

metastases or edema during prior therapy had a higher risk of death.

Across all lines of therapy, the median time to ramucirumab discontinuation was higher among patients who received combination therapy than those who received it as monotherapy (2.8 months [CI 2.4, 3.3] and 1.9 months [CI 1.4, 2.4], respectively; Fig. 3). There were no statistically significant differences in TTTD by line of therapy in which ramucirumab was initiated. Patients in the monotherapy subgroup with metastases to the peritoneum or liver were observed have an increased risk of treatment discontinuation (Table 3). In contrast, patients of Hispanic or Latino ethnicity were found to were observed to have a longer TTTD.

Several factors were found to be predictive of TTTD among patients treated with combination therapy (Table 3). Hispanic or Latino patients, non-White patients, as well as obese or overweight had lower risk of discontinuation. Those with hematological or pulmonary comorbidities, prior irinotecan-containing treatment and weight loss of over $10 \%$ were associated with increased risk of discontinuation.

\section{Predictive factors associated with monotherapy versus combination therapy}

Patients with prior use of a fluoropyrimidine-containing therapy were less likely to receive monotherapy (odds ratio [OR], 0.33; $P<0.0001$; Table 4). Initiation of ramucirumab by LOT was also a predictor of receiving monotherapy, and patients with an index date in the fourth-line setting were almost ten times more likely to receive monotherapy than patients who received it in the first-line setting (OR 9.82; $P<0.0047)$. Additionally, patients with an index date in LOT3 were $4.39(P=0.0244)$ times more likely to receive monotherapy compared to patients with first-line ramucirumab treatment. No other factors were consistently predictive of ramucirumab use, including age, gender, race, ethnicity, BMI, stage, comorbidities, histology, metastatic sites, toxicity or prior treatment.

\section{Discussion}

Real-world evidence has increasingly become a critical component of product development in a variety of therapeutic areas including oncology. There is increasing interest among providers, patients, industry, the FDA and other 


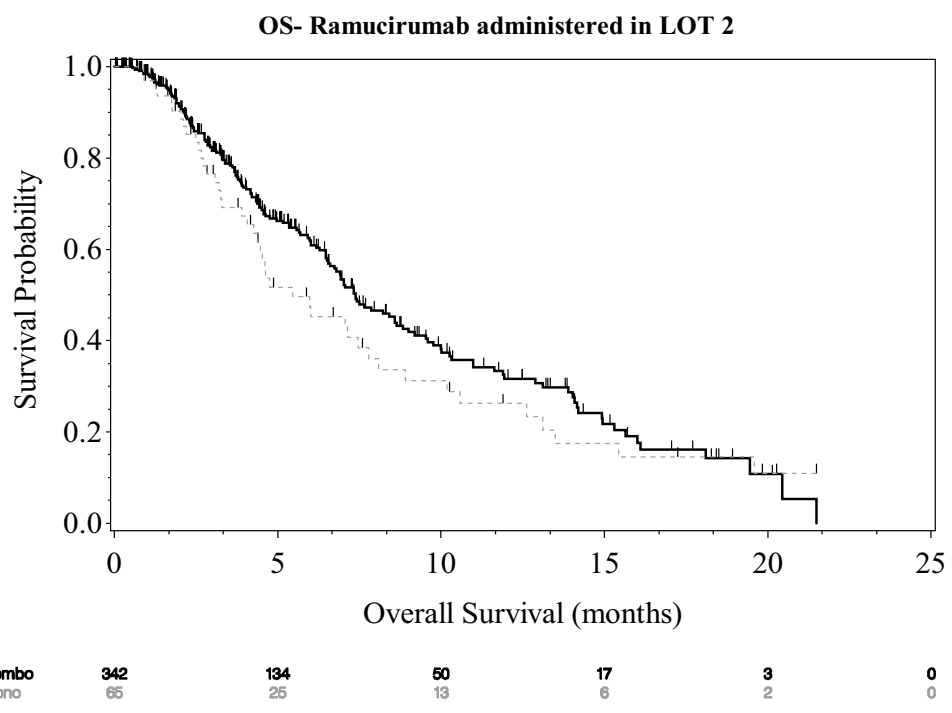

\begin{tabular}{|c|c|c|c|c|c|c|c|}
\hline \multirow[t]{2}{*}{ Variable } & \multirow[t]{2}{*}{ Level } & \multirow[t]{2}{*}{ \# of patients } & \multirow[t]{2}{*}{$\begin{array}{l}\text { Median survival } \\
\text { (95\% CI; months) }\end{array}$} & \multicolumn{4}{|c|}{ Survival rate $(95 \% \mathrm{CI})$} \\
\hline & & & & 6-month & 9-month & 12-month & 18-month \\
\hline \multirow[b]{2}{*}{ Group } & Monotherapy & 65 & $5.45(4.27,7.79)$ & $\begin{array}{c}47.44(33.60, \\
60.06)\end{array}$ & $\begin{array}{c}31.28 \\
(18.80,44.55) \\
\end{array}$ & $26.25(14.58,39.49)$ & $14.58(5.73,27.34)$ \\
\hline & Combination therapy & 342 & $7.40(6.60,8.84)$ & $\begin{array}{c}61.99(55.44 \\
67.86)\end{array}$ & $\begin{array}{c}42.56 \\
(35.43,49.49)\end{array}$ & $31.65(24.59,38.94)$ & $16.11(9.67,24.00)$ \\
\hline
\end{tabular}

CI, confidence interval; Combo, combination therapy; Mono, monotherapy

Fig. 2 Overall survival for patients receiving ramucirumab as monotherapy and combination therapy in the second-line setting

stakeholders to leverage real-world data outside of the traditional controlled, clinical trial setting to assist in the development of new drugs or expand labeled indications. Broader clinical effectiveness and safety data could impact quality, delivery of care and outcomes by accelerating our understanding of how to optimally incorporate treatments into everyday clinical practice. Importantly, real-world outcomes can also confirm the results of clinical trial data in the community practice setting. This has become particularly relevant for oncology treatments in a variety of tumor types, including gastric and GEJ cancers and treatments such as ramucirumab.

While the safety and efficacy of ramucirumab have been demonstrated in clinical trials, there is limited evidence of its use in real-world practice. To the best of our knowledge, this is the first study to examine patient characteristics, treatment patterns and clinical outcomes of ramucirumab for gastric or GEJ cancer in a community oncology setting. This information provides critical insight into providers' decision-making processes and, consequently, how ramucirumab is being used in realworld clinical practice. Moreover, this is the first study to demonstrate that clinical outcomes of patients receiving ramucirumab in the community oncology setting are comparable to those of patients in clinical trials.

In this study, the demographic and patient characteristics of gastric or GEJ cancer patients treated in the community setting appear to be similar to data reported in other clinical trials $[8,9]$. Overall survival estimates were also consistent with trends found in ramucirumab clinical trials. Among patients who initiated ramucirumab in the second-line setting, the median OS was 5.5 and 7.4 months for monotherapy and combination patients, respectively. In comparison, Fuchs et al. [10] found a median OS of 5.2 months among patients who received ramucirumab monotherapy in the REGARD clinical trial (over $90 \%$ of this patient population represented Western countries). Likewise, Wilke et al. [9] observed a median OS of 8.5 months among patients from North America, Australia, Israel and Europe who received ramucirumab in combination with paclitaxel in the RAINBOW clinical trial.

Predictors of OS and TTTD were explored through Cox proportional hazard analyses. Hispanic ethnicity was 
Table 3 Cox proportional hazard models for predictors of overall survival and time to treatment discontinuation

\begin{tabular}{|c|c|c|c|c|c|c|}
\hline Variable & Level & Hazard ratio & 95\% Lower CI & 95\% Upper CI & $\begin{array}{l}\text { Individual } \\
\text { effect } P \\
\text { value }\end{array}$ & $\begin{array}{l}\text { Overall } \\
\text { effect } P \\
\text { value }\end{array}$ \\
\hline \multicolumn{7}{|c|}{ Overall survival, monotherapy subgroup } \\
\hline \multirow[t]{2}{*}{ Ethnicity } & Not Hispanic or Latino (ref) & - & - & - & - & 0.0086 \\
\hline & Hispanic & 0.283 & 0.110 & 0.725 & 0.0086 & \\
\hline \multirow[t]{4}{*}{ Practice region } & South (reference) & - & - & - & - & 0.0071 \\
\hline & Midwest & 1.636 & 0.843 & 3.176 & 0.1459 & \\
\hline & Northeast & 0.142 & 0.032 & 0.628 & 0.0101 & \\
\hline & West & 0.685 & 0.380 & 1.234 & 0.2075 & \\
\hline \multirow[t]{2}{*}{ Liver metastasis } & No (reference) & - & - & - & - & 0.0523 \\
\hline & Yes & 1.674 & 0.995 & 2.818 & 0.0523 & \\
\hline \multirow[t]{2}{*}{ Peritoneal metastasis } & No (reference) & - & - & - & - & 0.0018 \\
\hline & Yes & 2.932 & 1.494 & 5.755 & 0.0018 & \\
\hline \multirow[t]{2}{*}{ Prior therapy toxicity: diarrhea } & No (reference) & - & - & - & - & 0.0541 \\
\hline & Yes & 0.423 & 0.177 & 1.015 & 0.0541 & \\
\hline \multicolumn{7}{|c|}{ Overall survival, combination therapy subgroup } \\
\hline \multirow[t]{3}{*}{ Race } & White (reference) & - & - & - & - & 0.0638 \\
\hline & Black & 0.509 & 0.264 & 0.982 & 0.0441 & \\
\hline & Other & 0.501 & 0.231 & 1.086 & 0.0800 & \\
\hline \multirow[t]{2}{*}{ Ethnicity } & Not Hispanic or Latino (reference) & - & - & - & - & 0.0014 \\
\hline & Hispanic or Latino & 0.468 & 0.294 & 0.745 & 0.0014 & \\
\hline \multirow[t]{4}{*}{ BMI status } & Normal (reference) & - & - & - & - & 0.0033 \\
\hline & Obese & 0.544 & 0.351 & 0.842 & 0.0063 & \\
\hline & Overweight & 1.086 & 0.731 & 1.613 & 0.6833 & \\
\hline & Underweight & 1.525 & 0.994 & 2.339 & 0.0531 & \\
\hline \multirow[t]{2}{*}{ ECOG status } & 0/1 (reference) & - & - & - & - & 0.0018 \\
\hline & $2+$ & 2.144 & 1.330 & 3.456 & 0.0018 & \\
\hline \multirow[t]{2}{*}{ Hematological comorbidities } & No (reference) & - & - & - & - & 0.0063 \\
\hline & Yes & 1.609 & 1.144 & 2.263 & 0.0063 & \\
\hline \multirow[t]{2}{*}{ Pulmonary comorbidities } & No (reference) & - & - & - & - & 0.0144 \\
\hline & Yes & 1.916 & 1.138 & 3.227 & 0.0144 & \\
\hline \multirow[t]{2}{*}{ Lung metastasis } & No (reference) & - & - & - & - & 0.0797 \\
\hline & Yes & 1.506 & 0.953 & 2.382 & 0.0797 & \\
\hline \multirow{2}{*}{$\begin{array}{l}\text { Prior fluoropyrimidine-contain- } \\
\text { ing treatment }\end{array}$} & No (reference) & - & - & - & - & 0.0791 \\
\hline & Yes & 1.365 & 0.965 & 1.931 & 0.0791 & \\
\hline \multirow[t]{2}{*}{ Prior therapy toxicity: edema } & No (reference) & - & - & - & - & 0.0132 \\
\hline & Yes & 2.059 & 1.163 & 3.646 & 0.0132 & \\
\hline \multicolumn{7}{|c|}{ Time to treatment discontinuation, monotherapy subgroup } \\
\hline \multirow[t]{2}{*}{ Ethnicity } & Not Hispanic or Latino (reference) & - & - & - & - & 0.0047 \\
\hline & Hispanic or Latino & 0.361 & 0.178 & 0.732 & 0.0047 & \\
\hline \multirow[t]{2}{*}{ Peritoneal metastasis } & No (reference) & - & - & - & - & 0.0059 \\
\hline & Yes & 2.041 & 1.229 & 3.392 & 0.0059 & \\
\hline \multirow[t]{2}{*}{ Liver metastasis } & No (reference) & - & - & - & - & 0.0283 \\
\hline & Yes & 1.613 & 1.052 & 2.472 & 0.0283 & \\
\hline \multicolumn{7}{|c|}{ Time to treatment discontinuation, combination therapy subgroup } \\
\hline Race & White (reference) & - & - & - & - & 0.0468 \\
\hline & Black & 0.791 & 0.481 & 1.299 & 0.3540 & \\
\hline & Other & 0.697 & 0.407 & 1.194 & 0.1887 & \\
\hline Ethnicity & Not Hispanic or Latino (reference) & - & - & - & - & $<0.0001$ \\
\hline & Hispanic or Latino & 0.484 & 0.341 & 0.687 & $<0.0001$ & \\
\hline
\end{tabular}


Table 3 (continued)

\begin{tabular}{|c|c|c|c|c|c|c|}
\hline Variable & Level & Hazard ratio & 95\% Lower CI & $95 \%$ Upper CI & $\begin{array}{l}\text { Individual } \\
\text { effect } P \\
\text { value }\end{array}$ & $\begin{array}{l}\text { Overall } \\
\text { effect } P \\
\text { value }\end{array}$ \\
\hline \multirow[t]{4}{*}{ BMI } & Normal (reference) & - & - & - & - & \multirow[t]{4}{*}{0.0003} \\
\hline & Obese & 0.638 & 0.455 & 0.894 & 0.0090 & \\
\hline & Overweight & 0.984 & 0.722 & 1.340 & 0.9177 & \\
\hline & Underweight & 1.563 & 1.120 & 2.181 & 0.0086 & \\
\hline \multirow[t]{3}{*}{ Weight loss } & $<10 \%$ (reference) & - & - & - & - & \multirow[t]{2}{*}{0.0383} \\
\hline & $\geq 10 \%$ & 2.356 & 1.047 & 5.302 & 0.0383 & \\
\hline & No weight loss vs. $<10 \%$ & 0.792 & 0.591 & 1.060 & 0.1171 & 0.1171 \\
\hline \multirow[t]{4}{*}{ Stage } & I (reference) & - & - & - & - & \multirow[t]{4}{*}{0.0300} \\
\hline & II & 1.559 & 0.747 & 3.253 & 0.2365 & \\
\hline & III & 1.685 & 0.815 & 3.484 & 0.1590 & \\
\hline & IV & 2.197 & 1.100 & 4.390 & 0.0258 & \\
\hline \multirow[t]{2}{*}{ Hematological comorbidities } & No (reference) & - & - & - & - & \multirow[t]{2}{*}{0.0872} \\
\hline & Yes & 1.277 & 0.965 & 1.691 & 0.0872 & \\
\hline \multirow[t]{2}{*}{ Pulmonary comorbidities } & No (reference) & - & - & - & - & \multirow[t]{2}{*}{0.0387} \\
\hline & Yes & 1.604 & 1.025 & 2.511 & 0.0387 & \\
\hline \multirow{2}{*}{$\begin{array}{l}\text { Prior antineoplastic-containing } \\
\text { treatment }\end{array}$} & No (reference) & - & - & - & - & \multirow[t]{2}{*}{0.0673} \\
\hline & Yes & 2.607 & 0.934 & 7.278 & 0.0673 & \\
\hline \multirow{2}{*}{$\begin{array}{l}\text { Prior irinotecan-containing } \\
\text { treatment }\end{array}$} & No (reference) & - & - & - & - & \multirow[t]{2}{*}{0.0313} \\
\hline & Yes & 1.628 & 1.045 & 2.537 & 0.0313 & \\
\hline \multirow{2}{*}{$\begin{array}{l}\text { Prior taxane-containing treat- } \\
\text { ment }\end{array}$} & No (reference) & - & - & - & - & \multirow[t]{2}{*}{0.0584} \\
\hline & Yes & 1.295 & 0.991 & 1.693 & 0.0584 & \\
\hline
\end{tabular}

$B M I$ body mass index, $C I$ confidence interval, ECOG Eastern Cooperative Oncology Group

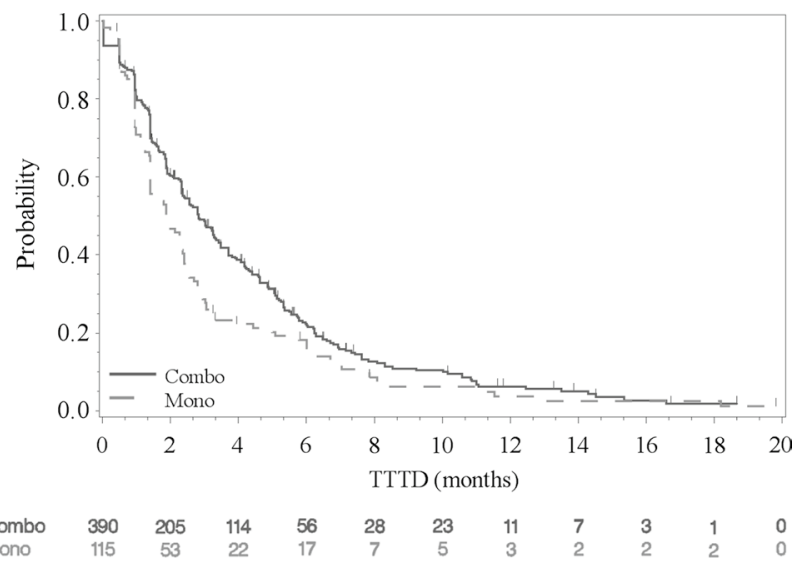

\begin{tabular}{|c|c|c|c|c|c|c|c|}
\hline \multirow[b]{2}{*}{ Variable } & \multirow[b]{2}{*}{ Level } & \multirow[b]{2}{*}{ \# of patients } & \multirow[t]{2}{*}{$\begin{array}{c}\text { Median DOT } \\
\text { (95\% CI; months) }\end{array}$} & \multicolumn{4}{|c|}{ Discontinuation rate $(95 \%$ CI) } \\
\hline & & & & 3- month & 6-month & 9-month & 12-month \\
\hline \multirow[b]{2}{*}{ Group } & Monotherapy & 115 & $1.87(1.41,2.37)$ & $71.29(62.75,79.34)$ & $81.76(73.96,88.38)$ & $93.87(87.75,97.56)$ & $96.32(92.47,99.51)$ \\
\hline & $\begin{array}{c}\text { Combination } \\
\text { therapy }\end{array}$ & 390 & $2.83(2.37,3.29)$ & $51.29(46.11,56.68)$ & $77.70(72.78,82.28)$ & $89.12(84.93,92.57)$ & $93.81(90.21,96.42)$ \\
\hline
\end{tabular}

DOT, duration of therapy; CI, confidence interval; Combo, combination therapy; Mono, monotherapy; TTTD, time to treatment discontinuation

Fig. 3 Time to ramucirumab treatment discontinuation among patients receiving ramucirumab as a monotherapy or combination therapy 
Table 4 Multivariable logistic regression for predictors of receiving ramucirumab as a monotherapy vs. combination therapy

\begin{tabular}{|c|c|c|c|c|c|c|c|}
\hline Variable & Level & $\begin{array}{l}N \text { (mono- } \\
\text { therapy } \\
\text { subgroup) }\end{array}$ & $\begin{array}{l}\text { Monotherapy } \\
\text { ramucirumab odds } \\
\text { ratio }\end{array}$ & $95 \%$ Lower CI & 95\% Upper CI & $\begin{array}{l}\text { Individual } \\
\text { effect } P \\
\text { value }\end{array}$ & $\begin{array}{l}\text { Overall } \\
\text { effect } P \\
\text { value }\end{array}$ \\
\hline \multirow{2}{*}{$\begin{array}{l}\text { Prior treatments for gastric or } \\
\text { GEJ cancer prior to ramu- } \\
\text { cirumab initiation: fluoropy- } \\
\text { rimidine }\end{array}$} & No (reference) & $171(66)$ & - & - & - & - & \multirow[t]{2}{*}{$<0.000$} \\
\hline & Yes & 334 (49) & 0.332 & 0.207 & 0.532 & $<0.0001$ & \\
\hline \multirow{4}{*}{$\begin{array}{l}\text { Line of therapy of ramucirumab } \\
\text { initiation }\end{array}$} & 1 (reference) & $15(4)$ & - & - & - & - & \multirow[t]{4}{*}{$<0.0001$} \\
\hline & 2 & $407(65)$ & 0.894 & 0.267 & 2.997 & 0.8565 & \\
\hline & 3 & $58(31)$ & 4.385 & 1.210 & 15.883 & 0.0244 & \\
\hline & $4+$ & $18(13)$ & 9.820 & 2.015 & 47.846 & 0.0047 & \\
\hline
\end{tabular}

CI confidence interval, GEJ gastroesophageal junction

associated with a lower risk of death and discontinuation for both the monotherapy and combination therapy subgroups. No other consistent predictors were identified in this study, although individual model results were similar to previous literature that reported performance status, liver and peritoneal metastases as prognostic factors [11, 12]. While several factors were statistically correlated with these clinical endpoints for the individual treatment subgroups (monotherapy vs. combination therapy), the clinical interpretations of these results are not definitive.

Examination of key demographic and clinical characteristics of study patients did not yield any unexpected predictors of monotherapy or combination therapy use. Instead, as anticipated, patients received ramucirumab in accordance with label indications. Following the November 2014 approval, combination therapy was received by more than $88 \%$ of all patients treated with ramucirumab. Patients previously treated with combination fluoropyrimidine- and platinum-containing regimens were more likely to receive ramucirumab monotherapy.

Expansion of the label indications during the study observation period are reflected by the differences in the proportion of patients receiving monotherapy or combination therapy. The overrepresentation of monotherapy that occurred during the April-November 2014 time period may have influenced the overall findings from this study, as both regimens were not FDA approved during this time.

These results demonstrate the real-world use of ramucirumab according to the label indications within the USON. In collaboration with the National Comprehensive Cancer Network (NCCN) and McKesson Specialty Health, the USON has developed treatment pathways to deliver evidence-based and standardized care to patients within the clinical workflow. The lack of other predictive factors associated with ramucirumab observed in this study, besides those supported by the label, suggests adherence to these treatment pathways. By decreasing variation in practice patterns and focusing on high-quality, cost-effective care, USON's treatment pathways have benefited patients' clinical outcomes.

The results of this study suggest the USON's iKM EHR provides a valuable source of real-world information about use of ramucirumab among a gastric or GEJ cancer patient population. This EHR system was designed by oncologists to advance the science of cancer care. With more than 975,000 patient records; 6800 concurrent users; and nearly 1000 provider users, the system provides insight into quality patient care and serves as a decision support tool at the point of care by providing staging and diagnosis suggestions according to criteria from the NCCN.

This was a retrospective assessment. The iKM data, despite its wealth of information about community-based oncology, is limited to clinics that are part of the USON; thus, results in this study cannot be generalized to the US population. There was the potential for documentation bias if there were omissions or errors. In addition, several key variables had a high proportion of missing structured data, which limits the conclusions that can be made.

\section{Conclusions}

Overall, the results confirm treatment pattern expectationsthat is, patients who received ramucirumab monotherapy did so prior to November 2014 and tended to be more heavily pre-treated than those who received it in combination. Moreover, based on these findings, it appears that clinical outcomes in the community oncology practices are similar to clinical outcomes as observed in clinical trials. The results of this study suggest the USON's iKM EHR provides a valuable source of real-world information about use of ramucirumab among a gastric or GEJ cancer patient population. Future research can expand upon these finding to explore other factors associated with use of ramucirumab and associated clinical outcomes. 


\section{Compliance with ethical standards}

Funding This study was funded Eli Lilly and Company.

Conflict of Interest Dr. Paulson has advisory board roles at Taiho, Merrimack, Bristol Myers Squibb, and Advanced Accelerator Application, as well as owns stock in Juno and Immunomedics. Dr. Hess is employed by Eli Lilly and Company. Dr. Liepa is employed by and owns stock in Eli Lilly and Company. Dr. Cui is employed by and owns stock in Eli Lilly and Company. Ms. Aguilar is employed by McKesson Specialty Health and provided research consulting services to Eli Lilly and Company. Ms. Clark is employed by McKesson Specialty Health, owns stock in McKesson and provided research consulting services to Eli Lilly and Company. Dr. Schelman is employed by and owns stock in Eli Lilly and Company.

Ethical standards Institutional Review Board and Compliance/Privacy approval was gained prior to initiation of the retrospective research. Since this project involved the analysis of existing data and records, study information was analyzed in such a manner that research participants could not be directly identified. Patient informed consent was not required due to the nature of the study design. Thus, exemption status and a waiver of informed consent were approved by The US Oncology, Inc. Institutional Review. Data was handled in compliance with HIPAA and Health Information Technology for Economic and Clinical Health (HITECH).

\section{References}

1. Siegel RL, Miller KD, Jemal A. Cancer Statistics, 2017. CA Cancer J Clin. 2017;67(1):7-30. https://doi.org/10.3322/caac.21387.

2. Karimi P, Islami F, Anandasabapathy S, Freedman ND, Kamangar F. Gastric cancer: descriptive epidemiology, risk factors, screening, and prevention. Cancer Epidemiol Biomark Prev. 2014;23(5):700-13. https://doi.org/10.1158/1055-9965. EPI-13-1057.

3. National Cancer Institute. Cancer stat facts: stomach cancer. December 20, 2016. https://seer.cancer.gov/statfacts/html/stoma ch.html.
4. National Comprehensive Cancer Network. NCCN clinical practice guidelines in oncology: gastric cancer, 2.20172017 8/1/17. https ://www.nccn.org/professionals/physician_gls/pdf/gastric.pdf.

5. Kanagavel D, Fedyanin M, Tryakin A, Tjulandin S. Second-line treatment of metastatic gastric cancer: current options and future directions. World J Gastroenterol. 2015;21(41):11621-35. https ://doi.org/10.3748/wjg.v21.i41.11621.

6. Eli Lilly and Company. Cyramza-package insert. 2015.

7. Oholendt AL, Zadlo JL. Ramucirumab: a new therapy for advanced gastric cancer. J Adv Pract Oncol. 2015;6(1):71-5.

8. Fuchs CS, Tomasek J, Yong CJ, Dumitru F, Passalacqua R, Goswami C, et al. Ramucirumab monotherapy for previously treated advanced gastric or gastro-oesophageal junction adenocarcinoma (REGARD): an international, randomised, multicentre, placebocontrolled, phase 3 trial. Lancet. 2014;383(9911):31-9. https:// doi.org/10.1016/S0140-6736(13)61719-5.

9. Wilke H, Muro K, Van Cutsem E, Oh SC, Bodoky G, Shimada Y, et al. Ramucirumab plus paclitaxel versus placebo plus paclitaxel in patients with previously treated advanced gastric or gastrooesophageal junction adenocarcinoma (RAINBOW): a doubleblind, randomised phase 3 trial. Lancet Oncol. 2014;15(11):1224 35. https://doi.org/10.1016/S1470-2045(14)70420-6.

10. De Vita F, Di Martino N, Fabozzi A, Laterza MM, Ventriglia J, Savastano B, et al. Clinical management of advanced gastric cancer: the role of new molecular drugs. World J Gastroenterol. 2014;20(40):14537-58. https://doi.org/10.3748/wjg.v20. i40.14537.

11. Fuchs CS, Muro K, Tomasek J, Van Cutsem E, Cho JY, Oh SC, et al. Prognostic factor analysis of overall survival in gastric cancer from two phase III studies of second-line ramucirumab (REGARD and RAINBOW) using pooled patient data. J Gastric Cancer. 2017;17(2):132-44. https://doi.org/10.5230/jgc.2017.17. e16.

12. Chau I, Norman AR, Cunningham D, Waters JS, Oates J, Ross PJ. Multivariate prognostic factor analysis in locally advanced and metastatic esophago-gastric cancer-pooled analysis from three multicenter, randomized, controlled trials using individual patient data. J Clin Oncol. 2004;22(12):2395-403. https://doi. org/10.1200/JCO.2004.08.154. 